\title{
Infecciones por Staphylococcus aureus resistente a meticilina adquiridas en la comunidad en niños antes sanos y en niños relacionados al hospital en la Argentina
}

\author{
Hugo Paganini, M. Paula Della L., Beatriz Muller O., Gustavo Ezcurra, Macarena Uranga, Clarisa Aguirre, \\ Gabriela Ensinck, Marys Kamiya, M. Rosa Miranda, Cristina Ciriaci, Claudia Hernández, Lidia Casimir, \\ M. José Rial, Norma Schenonne, Estela Ronchi, M. del Carmen Rodríguez, Fabiana Aprile, Catalina De Ricco, \\ Viviana Saito, Claudia Vrátnica, Laura Pons, Adriana Ernst, Sandra Morinigo, Marcelo Toffoli, Celia Bosque, \\ Victoria Monzani, Andrea Mónaco, José L. Pinheiro, M. del Pilar López, Leonardo Maninno y Claudia Sarkis
}

República Argentina, Ciudad Autónoma de Buenos Aires: Hospital Juan P. Garrahan (HP, MPDL, CH, LC, AM, JLP LM, CS) Hospital Pedro de Elizalde (BMO, MJR, NS, CDR)

Santa Fe: Hospital Alassia (GE, MRSA, ER, CB)

Buenos Aires: Hospital de Niños de San Justo (MU, MDCR, FA) Corrientes: Hospital Juan Pablo II (CA, VGS, CV) Rosario: Hospital de Niños J. Vilela (GE, AE)

Resistencia: Hospital Pediátrico Dr. Avelino L. Castelán (MKDM,

LP, SM)

Jujuy: Hospital de Niños Dr. Héctor Quintana (MRM, MT) Mar del Plata: Hospital Materno Infantil de Mar del Plata Don Victorio Tetamanti (CC, VM, MDPL).

Fuente de financiamiento: No hubo Los autores declaran no tener conflictos de interés

Recibido: 18 de enero de 2009 Aceptado: 24 de julio de 2009

Correspondencia a: Hugo Paganini hpaganini@intramed.net.ar

\section{Community onset of methicillin resistant Staphylococcus aureus infections in previously healthy or health care-associated children en Argentina}

Introduction: Community-acquired methicillin-resistant Staphylococcus aureus infections (CA-MRSA) are prevalent in several countries of the world. These infections seem to differ clinically from those occurring within the health care system (HCS-MRSA). Objective: To compare clinical characteristics of infections by CA-MRSA and HCA-MRSA in the same communitty. Material and Methods: Prospective, multicentric and comparative study. Children with clinically and microbiologicaly documented CA-MRSA were included. Results: Between $11 / 2006$ and 11/2007, 840 infections caused by $S$. aureus were diagnosed. Of them $582(68 \%)$ were communityacquired. Among these 356 (61\%) were CA-MRSA. In this group, 75 (21\%) were HCA-MRSA and $281(79 \%)$ CA-MRSA. The median age was 36 months (range: 1-201). Chronic skin disease (13) and chronic disease of CNS (9) were the underlying disease predominant. Children with CA-MRSA had more frequency of previous antibiotic treatment (63 vs 34\%) and previous medical consult (76 vs 52\%), invasive procedures (31vs 8\%), surgery (15 vs $0,3 \%)$ and fever $(94$ vs $74 \%)(\mathrm{p}=<.05)$. Children with CA-MRSA had subcutaneous abscesses $(34$ vs $15 \%)$ $(\mathrm{p}=<.05)$ more frequently. Bacteremia and sepsis rate was similar in both groups $(21 \mathrm{vs} 18 \%$ and $17 \mathrm{vs} 11 \%$ respectively) $(\mathrm{p}=\mathrm{NS})$. Antibiotic resistance was more frequent in children with HCA-MRSA: Rifampin (7 vs $1 \%)$, trimethoprim-sulphametoxazole (7vs 1\%) and clindamycin $(25 v s 9 \%)(\mathrm{p}=<.05)$. Four children $(5 \%)$ with HCA-MRSA infections died and $3(1 \%)$ in CA-MRSA group $(\mathrm{p}=.05)$. Conclusion: Children with HCA-MRSA infections more frequent antibiotic resistance than CA-MRSA should be reconsider the empiric antibiotic treatment of community-acquired infections in children in our area.

Key words: Community-acquired, Staphylococcus aureus, methicillin-resistant, children

Palabras clave: Comunitario, Staphylococcus aureus, meticilina-resistente, niños.

\section{Introducción}

S taphylococcus aureus resistente a meticilina (SAMR) es un patógeno frecuente en niños hospitalizados ${ }^{1}$. Causa una amplia gama de infecciones desde la leves (ej. infecciones localizadas de piel) hasta las graves (ej. sepsis) ${ }^{1-3}$. Un nuevo tipo de $S$. aureus resistente a meticilina que infecta a personas provenientes de la comunidad ha aparecido en los últimos años en diversas regiones del planeta ${ }^{4}$. Estas cepas presentan características microbiológicas particulares. La resistencia a todos los antimicrobianos $\beta$-lactámicos es mediada por un gen denominado mec $A$ que codifica la síntesis de una proteína de unión a penicilina (PBP2A) de la bacteria ${ }^{5}$. Este gen se encuentra localizado en una isla genética llamada cassette cromosómico mec (SCCmec). Las cepas adquiridas en la comunidad llevan el SCCmec IV a diferencia de las circulantes dentro del hospital donde prevalecen del I al III ${ }^{5,6}$. Tienen un patrón de susceptibilidad a antimicrobianos diferente a las de origen hospitalario. Demuestran solamente resistencia a meticilina y buen nivel de susceptibilidad a clindamicina, cotrimoxazol y tetraciclinas ${ }^{5}$. Provocan infecciones de la piel preferentemente $(85-90 \%$ de los casos) y en $\sim 10 \%$ causan infecciones graves (ej. sepsis o neumonía necrosante más frecuentemente) que pueden ocasionar la muerte del paciente ${ }^{4,5,7,8}$.

En un estudio llevado a cabo recientemente en E.U.A., se pudo determinar que la tasa de infección por S. aureus resistente a meticilina de origen comunitario (SAMRCO) 
en ese país osciló entre 16 y 70 casos por 100.000 en niños bajo 5 años de edad 9 .

Los primeros casos en Latinoamérica fueron reportados por Uruguay en el año $2003^{10}$. En el año 2005 nuestro grupo de trabajo informó que la prevalencia de infecciones por SAMRCO en Argentina era de $42 \%{ }^{11}$. Esta cifra aumentó durante el año 2006 a $51 \%$ en una serie de 87 niños analizados ${ }^{12}$.

Las infecciones por SAMRC que se presentan en niños con enfermedades de base o que concurren en forma asidua a los hospitales para controles médicos u hospitalizaciones conforman un grupo con características diferentes a aquellas que adquieren los niños antes $\operatorname{sanos}^{5,13,14}$. Muy pocos estudios han valorado este tipo de infecciones en niños ${ }^{11}$, y en nuestro medio no existen reportes al respecto.

En base a lo expuesto precedentemente realizamos un estudio multicéntrico y prospectivo de las infecciones causadas por SAMRCO con el objetivo de comparar aquellas que se presentaban en los niños sanos con las producidas en niños con enfermedad de base o que habían concurrido en forma frecuente a centros de salud.

\section{Material y Métodos}

Tipo de estudio. Se realizó un estudio prospectivo y multicéntrico en nueve diferentes centros de la Argentina: Ciudad Autónoma de Buenos Aires (dos centros), San Justo, Provincia de Buenos Aires, Mar del Plata, Rosario, Santa Fe, Corrientes, Resistencia y Jujuy. Se efectuó un análisis comparativo entre las infecciones causadas por $S a M R C$ que padecían los niños con enfermedad de base o que tenían el antecedente de consultas previas o internación en hospitales con niños antes sanos, con el mismo tipo de infección.

Los Comités de Investigación de cada hospital revisaron y aprobaron el estudio antes de su comienzo.

Criterio de inclusión. Fueron incluidos todos los niños que presentaron una infección clínica y microbiológicamente documentada por SAMR sobre un mes de vida.

Definiciones. Las infecciones fueron categorizadas en:

- Infección proveniente de la comunidad en un niño antes sano (SAMR-CO): Fue aquella que era adquirida en la comunidad y diagnosticada en las primeras 48 horas de la internación o cuando el diagnóstico era realizado en forma ambulatoria en un niño previamente sano, sin antecedentes de internación en el año anterior a la consulta ${ }^{4}$.

- Infección proveniente de la comunidad en niños con enfermedad de base o con consulta o internación previa en el hospital (SAMRC-RH): Se la definió si ocurría en un niño que tenía alguna patología de base que condicionara la concurrencia asidua a un centro asistencial para atención de su enfermedad (Ej. fibrosis quística, enfermedades crónicas de la piel, del metabolismo, cardíacas o neurológicas, cáncer, etc) o si había sido internado en el año previo y dado de alta hacía más de 72 horas $^{11}$.

Tratamiento antimicrobiano. Fue considerado como discordante si el antimicrobiano indicado no era efectivo en base al antibiograma del patógeno hallado. Si el niño recibía más de un antimicrobiano en el tratamiento empírico y uno de los mismos era útil para el patógeno hallado, se le consideró concordante.

Evolución. Los pacientes fueron evaluados hasta 30 días tras finalizar el tratamiento de la infección para calcular la mortalidad. La muerte fue atribuida a la infección si el niño tenía hemocultivos positivos en el momento del deceso o si tenía signos persistentes de infección sistémica refractarios al tratamiento instituido (ej. fiebre, leucocitosis, hipotensión arterial, etc) en el momento de la muerte.

Microbiología. Se identificó a la especie $S$. aureus por pruebas microbiológicas habituales. La susceptibilidad a meticilina se determinó mediante pruebas de difusión con discos de oxacilina de $1 \mu \mathrm{g}$ y de cefoxitina de $30 \mu \mathrm{g}$, en medio agar de Mueller Hinton (MHA) con incubación a $37^{\circ} \mathrm{C}$ durante 24 horas. La detección de halos menores de $20 \mathrm{~mm}$ se consideró como resistencia. Se realizó detección selectiva en placa de MHA con $4 \%$ de cloruro de sodio y 6 $\mu \mathrm{g} / \mathrm{ml}$ de oxacilina, con inóculo de 100.000 ufc. Toda cepa que se desarrolló fue considerada meticilina-resistente ${ }^{15}$.

Análisis estadístico. Los datos numéricos se expresaron como medias aritméticas y se compararon utilizando pruebas paramétricas ( $\mathrm{T}$ test de Student) para las de distribución normal, y no paramétricos (Wilcoxon) para las variables de tiempo con distribución asimétrica. Un valor de $\mathrm{p}<$ de 0,05 se consideró significativo. Todos los datos se almacenaron en un programa de computación con el software Epi-info versión 2000 (CDC, Atlanta, Georgia, E. U.A.).

\section{Resultados}

Entre los meses de noviembre de 2006 y noviembre de 2007 fueron diagnosticadas 840 infecciones por $S$. aureus. De ellas 582 (69\%) fueron adquiridas en la comunidad. Dentro de estas últimas $356(61 \%)$ fueron causadas por SAMRC, de las cuales 75 (21\%) fueron SAMRC-RH y $281(79 \%)$ con SAMR-CO. Veintidós infecciones (SAMRC-RH: 3 y SAMR-CO: 19) no fueron incluidos en el estudio por dificultades en la localización del paciente. 


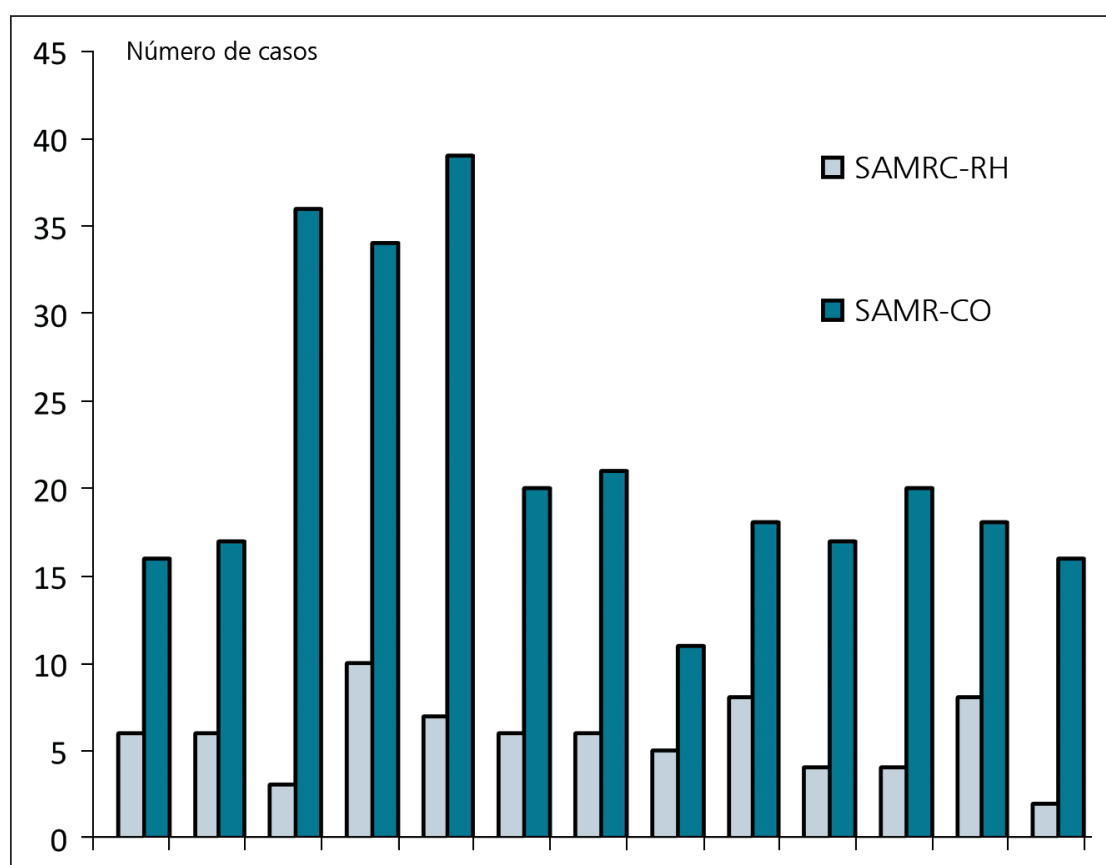

Nov Dic Ene Feb Mar Abr May Jun Jul Ago Set Oct Nov

SaMRC-RH: Staphylococcus aureus meticilina-resistente relacionado con el hospital $(n=75)$

SaMR-CO: Staphylococcus aureus meticilina-resistente de la comunidad en niños antes sanos $(\mathrm{n}=281)$

Figura 1. Distribución de las infecciones causadas por Staphylococcus aureus resistente a meticilina provenientes de la comunidad, de acuerdo al mes del año $(n=356)$.

Tabla 1. Distribución de las infecciones por Staphylococcus aureus resistente a meticilina provenientes de la comunidad, de acuerdo al centro

\begin{tabular}{|lcc|}
\hline Centro & $\begin{array}{c}\text { SAMR (n= 356) } \\
\text { SAMR-Co } \\
\text { (n = 281) }\end{array}$ & $\begin{array}{c}\text { SAMRC-RH } \\
(\mathbf{n}=\mathbf{7 5})\end{array}$ \\
CABA 1 & 53 & 18 \\
CABA 2 & 42 & 16 \\
Santa Fe & 48 & 8 \\
San Justo (Buenos Aires) & 33 & 10 \\
Corrientes & 28 & 5 \\
Resistencia (Chaco) & 24 & 9 \\
Rosario (Santa Fe) & 29 & 2 \\
Jujuy & 10 & 4 \\
Mar del Plata (Buenos Aires) & 14 & \\
\hline CABA: Ciudad Autónoma de Buenos Aires. Valores expresados en número absoluto de casos. \\
SaMRC-RH: Staphylococcus aureus meticilina-resistente relacionado con el hospital o con enfermedad \\
de base. SaMR-CO: Staphylococcus aureus meticilina-resistente proveniente de la comunidad en niños \\
antes sanos.
\end{tabular}

La distribución anual de las infecciones pueden observarse en la Figura 1, donde destaca el predominio de las mismas en los meses de verano y primavera. En la Tabla 1 se muestra la distribución de los casos de acuerdo al centro, pudiéndose apreciar que no hubo diferencias significativas en el número de casos según la institución.

Las enfermedades de base que más frecuentemente presentaron los niños fueron: enfermedades crónicas de la piel, patología crónica del SNC, infección por el $\mathrm{VIH}$, afecciones cardiovasculares e inmunodeficiencias (Tabla 2).

La media de edad de los niños fue semejante en ambos grupos. No se observaron diferencias estadísticamente significativas en la distribución por sexo, número de contactos domiciliarios, tener cobertura médica, concurrencia a guardería y la presencia de trabajadores de la salud en la familia $(\mathrm{p}=\mathrm{NS})$. Los niños con infección por SAMRC-RH había recibido con mayor frecuencia tratamiento antimicrobiano previo, procedimientos invasores y cirugía antes de adquirir la infección $(\mathrm{p}<0,05)$ (Tabla 3$)$.

El 93\% (70) de los niños con SAMRC-RH presentaron foco clínico de infección al ingreso mientras que $99 \%$ (280) de los pacientes antes sanos lo padecían $(\mathrm{p}<0,05)$. Prevalecieron las infecciones de piel y tejidos blandos en ambos grupos de pacientes. Pudo observarse mayor tasa de abscesos subcutáneos en los niños con infección por SAMR-CO $(\mathrm{p}<0,05)$ (Tabla 4).

Tabla 2. Tipo de enfermedad de base de niños con infección por Staphylococcus aureus resistente a meticilina proveniente de la comunidad $(n=75)$

\begin{tabular}{lcc} 
Tipo de enfermedad de base & $\mathbf{n}$ & (\%) \\
Enfermedades crónicas de la piel & 13 & $(18)$ \\
Enfermedad crónica del SNC & 9 & $(12)$ \\
\hline Infección por VIH & 7 & $(9)$ \\
\hline Cardiopatías & 6 & $(8)$ \\
\hline Inmunodeficiencias congénitas & 6 & $(8)$ \\
Cáncer & 2 & $(3)$ \\
Afecciones metabólicas & 1 & $(1)$ \\
Enfermedad pulmonar crónica & 1 & $(1)$ \\
\hline Trasplante hepático & 1 & $(1)$ \\
Insuficiencia renal crónica & 1 & $(1)$ \\
\hline Otros & 28 & $(38)$ \\
\hline
\end{tabular}

SNC: sistema nervioso central. VIH: virus de inmunodeficiencia humana. 


\begin{tabular}{|c|c|c|}
\hline Variable & $\begin{array}{l}\text { SAMRC-RH } \\
(\mathrm{n}: 75)\end{array}$ & $\begin{array}{l}\text { SAMR-CO } \\
\text { (n: 281) }\end{array}$ \\
\hline Edad media en meses & 54 & 57,9 \\
\hline Sexo masculino: $n(\%)$ & $46(61)$ & $167(59)$ \\
\hline $\mathrm{N}^{\circ}$ de contactos domiciliarios (media y rango) & $5,3(2-10)$ & $5,2(1-10)$ \\
\hline Cobertura médica: n (\%) & $5(7)$ & $30(1)$ \\
\hline Concurrencia a la guardería: n (\%) & $3(4)$ & $12(4)$ \\
\hline Trabajador de la salud en el domicilio: $n(\%)$ & $2(3)$ & $11(4)$ \\
\hline Tratamiento antimicrobiano previo: $\mathrm{n}(\%)$ & $47(63)$ & $94(33)^{*}$ \\
\hline Procedimiento invasivo previo: $\mathrm{n}(\%)$ & $23(31)$ & $23(8)^{*}$ \\
\hline Cirugía previa: n (\%) & $11(15)$ & $3(1)^{*}$ \\
\hline \multicolumn{3}{|c|}{$\begin{array}{l}\text { SaMRC-RH: Staphylococcus aureus meticilina-resistente relacionado con el hospital. SaMR-CO: Sta- } \\
\text { phylococcus aureus meticilina-resistente de la comunidad en niños antes sanos. }{ }^{*} p<0,05 \text {. }\end{array}$} \\
\hline
\end{tabular}

Tabla 4. Focos clínicos iniciales de infección más frecuentes en niños con infección por $S$. aureus resistente a meticilina adquirida en la comunidad, en ambos grupos de pacientes

\begin{tabular}{|c|c|c|}
\hline Tipo de foco clínico & $\begin{array}{c}\text { SAMRC-RH } \\
(\mathrm{n}: 70)\end{array}$ & $\begin{array}{l}\text { SAMR-CO } \\
\text { (n: 280) }\end{array}$ \\
\hline Celulitis: n (\%) & $25 \quad(36)$ & $85 \quad(30)$ \\
\hline Abscesos subcutáneos: n (\%) & 11 (16) & $96(34)^{*}$ \\
\hline Osteomielitis: n (\%) & $3 \quad(4)$ & $23 \quad(8)$ \\
\hline Artritis: n (\%) & $4 \quad(6)$ & $21 \quad(7)$ \\
\hline Empiema pleural: $n(\%)$ & $3 \quad(4)$ & $19 \quad(7)$ \\
\hline Neumonía: n (\%) & $5 \quad(7)$ & $16 \quad(6)$ \\
\hline Impétigo: n (\%) & $5 \quad(7)$ & $13 \quad(5)$ \\
\hline Absceso de psoas: n (\%) & $2 \quad(3)$ & $6 \quad(2)$ \\
\hline Piomiositis: n (\%) & $3 \quad(4)$ & $4 \quad(1)$ \\
\hline \multicolumn{3}{|c|}{$\begin{array}{l}\text { SaMRC-RH: Staphylococcus aureus meticilina-resistente relacionado } \\
\text { con el hospital. SaMR-CO: Staphylococcus aureus meticilina-resis- } \\
\text { tente de la comunidad en niños antes sanos. }{ }^{*} p<0,05 \text {. }\end{array}$} \\
\hline
\end{tabular}

El 21\% (16) de los pacientes infectados con SAMRC$\mathrm{RH}$ y $18 \%$ (51) de los sanos tuvieron bacteriemia ( $\mathrm{p}=$ NS). Solamente los pacientes infectados con SAMRCRH presentaron bacteriemia asociada a catéter $(5 ; 7 \%)$. La fiebre estuvo presente en 61\% (46) de los niños con SAMRC-RH y en 74\% (208) de los pacientes con SAMRCO $(\mathrm{p}<0,05)$. No hubo diferencias en la duración del cuadro febril entre los dos grupos (Media: 3,3 y 3,4 días en niños con SAMRC-RH y SAMR-CO, respectivamente). El 17\% (13) de los niños con SAMRC-RH presentó sepsis, mientras que $11 \%$ (30) de los sanos padeció este cuadro $(\mathrm{p}=\mathrm{NS})$.

Se pudo diagnosticar un foco secundario en $8 \%$ (6) de los niños con infección con SAMRC-RH y en 10\% (28) de los pacientes con SAMR-CO ( $\mathrm{p}=\mathrm{NS})$. La osteomielitis fue el foco secundario más frecuente en ambos grupos, prevaleciendo en los niños con SAMRC-RH 4 (70\%) sobre los niños antes sanos $10(36 \%)(\mathrm{p}<0,05)$.

Los niños infectados con cepas de SAMRC-RH tuvieron menos tasa de leucocitosis que los pacientes antes sanos $\left(13.600 / \mathrm{mm}^{3}\right.$ versus $\left.15.000 / \mathrm{mm}^{3}\right)(\mathrm{p}<0,05)$.

Las cepas aisladas de pacientes con $\mathrm{SaMRC-RH}$ presentaron con mayor frecuencia resistencia a rifampicina, gentamicina, ciprofloxacina, cotrimoxazol, clindamicina y eritromicina $(\mathrm{p}<0,05)$. La resistencia a minociclina fue semejante en ambos grupos de pacientes (Tabla 5).

La mayor parte de los niños recibieron tratamiento antimicrobiano empírico ( $92 \%$ de niños con SAMRC-RH versus $98 \%$ de los niños antes sanos) $(p=N S)$. No se ob- servaron diferencias en la tasa de tratamiento discordante, ni en el tiempo de retraso del tratamiento entre ambos grupos de pacientes. La duración del tratamiento parenteral fue semejante en ambos grupos (media de 9,9 días en niños con SAMRC-RH versus 9,2 días en niños antes sanos) $(\mathrm{p}=\mathrm{NS})$. Los pacientes con infección por SAMR-

\begin{tabular}{|c|c|c|}
\hline \multicolumn{3}{|c|}{$\begin{array}{l}\text { Tabla 5. Resistencia a antimicrobianos de las } \\
\text { cepas de SAMRC aisladas de ambos grupos de } \\
\text { pacientes. Valores expresados en números } \\
\text { absolutos de tasa de resistencia sobre cepas } \\
\text { testeadas y porcentajes de cepas resistentes }\end{array}$} \\
\hline Antimicrobiano & $\begin{array}{l}\text { SAMRC-RH } \\
\text { (n: 70) }\end{array}$ & $\begin{array}{l}\text { SAMR-CO } \\
\text { (n: 281) }\end{array}$ \\
\hline Rifampicina: $\quad$ n/total (\%) & $5 / 75 \quad(7)$ & $3 / 273 \quad(1)^{*}$ \\
\hline Gentamicina: n/total (\%) & $16 / 71(22)$ & $19 / 252 \quad(7)^{*}$ \\
\hline Ciprofloxacina: n/total (\%) & $3 / 62 \quad(5)$ & $2 / 241 \quad(1)^{*}$ \\
\hline Cotrimoxazol: $\mathrm{n} /$ total (\%) & $5 / 73 \quad(7)$ & $2 / 272 \quad(1)^{*}$ \\
\hline Clindamicina: n/total (\%) & 19/73 (26) & $26 / 273 \quad(9)^{*}$ \\
\hline Eritromicina: $\quad \mathrm{n} /$ total (\%) & $21 / 70(30)$ & $34 / 274(12)^{*}$ \\
\hline Minociclina: $\quad$ n/total (\%) & $2 / 60$ & $1 / 219 \quad(0,4)$ \\
\hline
\end{tabular}


Tabla 6. Evolución comparativa de los niños con infección por Staphylococcus aureus resistente a meticilina adquirido en la comunidad

\begin{tabular}{|lcc|} 
Variable & $\begin{array}{c}\text { SAMRC-RH } \\
(\mathbf{n}=\mathbf{7 0})\end{array}$ & $\begin{array}{c}\text { SAMR-CO } \\
(\mathbf{n}=\mathbf{2 8 1})\end{array}$ \\
Tratamiento discordante: $\mathrm{n}(\%)$ & $43(57)$ & $195(72)$ \\
Retraso en el tratamiento apropiado: $\mathrm{n}(\%)$ & $21(30)$ & $69(24)$ \\
Tiempo de retraso del tratamiento apropiado (horas) & 96 & 72 \\
Cambio al tratamiento empírico: $\mathrm{n}(\%)$ & $27(36)$ & $106(37)$ \\
Duración del tratamiento parenteral (días) & 9,9 & 9,2 \\
Duración del tratamiento oral (días) & 10,4 & 12,8 \\
Tratamiento quirúrgico: $\mathrm{n}(\%)$ & $32(43)$ & $203(72)$ * \\
Complicación $\mathrm{n}(\%)$ & $5(7)$ & $17(6)$ \\
Fallecidos & $4(5)$ & $3(1)$ \# \\
\hline
\end{tabular}

SaMRC-RH: Staphylococcus aureus meticilina-resistente relacionado con el hospital SaMR-CO: Staphylococcus aureus meticilina-resistente de la comunidad en niños antes sanos ${ }^{*} p<0,05 ; \# p=0,05$. informados son altamente representativos de la geografía de nuestro país y nos muestran que la tasa de resistencia a meticilina en los aislados de $S$. aureus provenientes de la comunidad es mayor al $50 \%$ en todos los centros.

En el año 2005 Hultén y colaboradores reportaron sobre-infecciones por SAMR-C que afectaban a pacientes que presentaban enfermedades de base o que habían sido internados en forma previa ${ }^{14}$. Estos niños concurrían en forma asidua a los hospitales o a las consultas médicas debido a su enfermedad de base. Si bien las infecciones estafilocóccicas en estos niños cumplían con el requisito de haber sido adquiridas fuera del hospital, su comportamiento clínico y fundamentalmente, su tasa de resistencia a los antimicrobianos era diferente ${ }^{13,14}$.

Las enfermedades de base más frecuentes halladas en nuestros pacientes fueron las enfermedades de la piel, seguidas de las del SNC y la infección por VIH. Este hallazgo es semejante a lo publicado previamente, excepto que las enfermedades crónicas del pulmón no prevalecieron en nuestros niños, a diferencia de lo reportado por Hultén en su serie de pacientes ${ }^{14}$.

Las infecciones de piel y tejidos blandos son las formas clínicas de presentación más frecuentes de las infecciones por SAMRC ${ }^{4,5}$. La celulitis, la forunculosis y los abscesos subcutáneos son los cuadros predominantes. En la serie de pacientes aquí reportados, se pudo observar que los niños con infección por SAMR-CO presentaban, con mayor frecuencia, abscesos subcutáneos que aquellos que tenían alguna patología de base. Cabe recordar que en las series internacionales de pacientes con infección causada por SAMRC en quienes no se observaba una enfermedad de base definida o no tenían el antecedente de haber estado hospitalizados, los abscesos subcutáneos fueron las formas clínicas prevalentes ${ }^{4,5}$.

Cotrimoxazol, vancomicina y clindamicina son antimicrobianos útiles para el tratamiento de estas infecciones. La tasa de resistencia a cotrimoxazol en cepas de SAMRC es baja ${ }^{4,5,9}$. Se ha publicado una tasa de resistencia variable a clindamicina. En el Hospital de Niños de Texas, en E.U.A., ésta llegó a $10 \%$ en los pacientes con cepas de SAMS y a $18 \%$ en las halladas de niños con SAMRC-RH ${ }^{4}$. La tasa de resistencia en nuestros niños con infección por SAMRC-RH fue mayor a aquellos que no la tenían (26 versus $9 \%$ ). Este es un dato muy importante, ya que según la recomendación de expertos, clindamicina es un antimicrobiano de utilidad para el tratamiento empírico de estas infecciones, salvo que la tasa de resistencia sea mayor al $15 \%$ en el área de trabajo ${ }^{5}$. Según los datos mostrados precedentemente, no debería utilizarse clindamicina como tratamiento empírico en niños con infecciones producidas por cepas de SAMRC-RH en nuestro país.

El tratamiento quirúrgico es muy importante para garantizar la cura de las infecciones de piel localizadas (ej. abscesos subcutáneos $)^{4}$. Lee y cols, reportaron una serie 
de 69 niños con infecciones de piel y tejidos blandos por SAMRC donde pudieron demostrar que el tratamiento quirúrgico fue la base fundamental para garantizar la cura de las infecciones, independientemente del antimicrobiano administrado ${ }^{24-26}$. En la serie de niños presentados aquí se muestra que en los pacientes con SAMR-CO, que presentan con mayor frecuencia infecciones localizadas de la piel, tienen una mayor tasa de indicación de cirugía.

Los pacientes que presentaron infección por SAMRC y con SAMRC-RH, tuvieron una frecuencia alta de tratamiento discordante al momento del ingreso. Esta diferencia no fue estadísticamente significativa entre los dos grupos de estudio, pero igualmente fue mayor en el grupo de pacientes con SAMR-CO. Probablemente esto se deba a que los niños que tuvieron SAMRC-RH recibieron tratamiento antimicrobiano de amplio espectro y frecuentemente combinado, a diferencia de los niños sanos. El tiempo de retraso para realizar un tratamiento concordante fue alto en los dos grupos de estudio (entre 72 y 96 horas). Este es un punto muy importante a tener en cuenta, ya que plantea la necesidad de discutir el tipo y momento de la indicación de cobertura frente a SAMRC en niños para disminuir este tiempo de retraso y de esta manera mejorar la evolución de estas infecciones.

No hay estudios suficientes que hayan analizado la letalidad de estas infecciones en niños ${ }^{4,9}$. El análisis de los pacientes incluidos en este reporte permitió determinar que la mortalidad en los niños con SAMRC-RH fue mayor que aquellos con SAMR-CO $(p=0,05)$. La presencia de una patología de base, como se puede observar, agravaría la evolución de estas infecciones. Igualmente, el número de pacientes fallecidos fue pequeño, lo que no permite sacar una conclusión definitiva respecto a este punto y no se puede aseverar que estas infecciones presenten peor evolución que las causadas por SAMR-CO.

Cabe mencionar que este estudio presenta limitaciones: la tasa real de infecciones de piel y tejidos blandos no pudo precisarse en forma exacta, ya que no todos los niños con infecciones de piel atendidos en los hospitales fueron sometidos a la toma de muestra para estudio microbiológico; la poca cantidad de pacientes fallecidos no permitió tomar conclusión sobre el impacto de estas infecciones en la mortalidad.

En suma destacamos:

- La tasa de infecciones causadas por SAMRC es alta en niños de nuestro país. Este es un alerta epidemiológico, particularmente para los pediatras.

- Los niños con SAMRC-RH tienen menor tasa de infección localizada de piel y tejidos blandos y mayor mortalidad.
- La tasa de resistencia a los antimicrobianos es mayor en los niños con SAMRC-RH.

- La resistencia a clindamicina supera el $15 \%$ en los niños con SAMRC-RH. Esto permite afirmar que no se debe utilizar este antimicrobiano ante la sospecha de estas infecciones en niños con patología de base, que concurran asiduamente a los hospitales o que hayan sido hospitalizados en el año previo.

- Debe revisarse y normarse en forma urgente el tratamiento empírico de las infecciones provenientes de la comunidad en niños.

\section{Resumen}

Introducción: Staphylococcus aureus meticilinaresistente proveniente de la comunidad (SAMRC) es altamente prevalente en diversos países del planeta. Objetivos: Realizar un análisis clínico comparativo entre las infecciones por SAMRC en niños antes sanos (SAMR-CO) y aquellos con S. aureus MR en pacientes con patologías previas (SAMR-RH). Material y Métodos: Estudio multicéntrico, prospectivo y comparativo. Fueron incluidos los niños que tenían infección clínica y microbiológicamente documentada por SAMRC. Resultados: Entre 11/2006 y 11/2007 fueron diagnosticadas 840 infecciones por $S$. aureus. De ellas $582(69 \%)$ fueron detectadas en la comunidad. Entre estas $356(61 \%)$ fueron SAMRC. Entre estas últimas 75 (21\%) fueron SAMR-RH y 281 (79\%) SAMR-CO. La mediana de edad fue de 36 meses (rango: 1-201). Las enfermedades de base más frecuentes fueron: dermatopatías crónicas (13) y enfermedad crónica del SNC (9). Los niños con infección por SAMR-RH presentaron con mayor frecuencia tratamiento antimicrobiano previo (63 vs 34\%), consultas médicas previas (76 vs 52\%), procedimiento invasor previo (31 vs $8 \%$ ), cirugía (15 vs $0,3 \%$ ) y fiebre al momento de la consulta $(94$ vs $74 \%)(\mathrm{p}<0,05)$. Los niños con infección por SAMR-CO tuvieron con mayor frecuencia abscesos subcutáneos (34 vs 15\%) $(\mathrm{p}<0,05)$. La tasa de bacteriemia y se sepsis fue semejante en ambos grupos (21 vs 18\% y 17 vs 11\%) ( $\mathrm{p}=\mathrm{NS})$. La resistencia a antimicrobianos fue mayor en niños con SAMR-RH: rifampicina (7 vs $1 \%$ ), cotrimoxazol (7vs 1\%) y clindamicina (25 vs 9\%) $(p<0,05)$. Fallecieron 4 niños con SAMR-RH $(5 \%)$ y 3 niños con SAMR-CO $(1 \%)(p=0,05)$. Conclusión: Los niños con SAMR-RH presentan mayor tasa de resistencia a antimicrobianos que SAMR-CO. Debe replantearse el tratamiento antimicrobiano empírico en niños con infecciones provenientes de la comunidad en nuestro medio. 


\section{Referencias}

1.- Paganini H. Infecciones por Staphylococcus aureus en: Paganini H., Infectología Pediátrica, Ed. Científica Interamericana, 2007, pág 95562.

2.- Benner E J, Kayser F H. Growing clinical significance of methicillin-resistant Staphylococcus aureus. Lancet 1968; 2:741-4.

3.- Barrett F F, McGehee R F Jr, Finland M. Methicillin-resistant Staphylococcus aureus at Boston City Hospital. Bacteriologic and epidemiologic observations. N Engl J Med 1968; 279: 441-8.

4.- Kaplan S L, Hulten K G, González B E, Hammerman W A, Lamberth L, Versalovic J, et al. Three-year surveillance of communityacquired Staphylococcus aureus infections in children. Clin Infect Dis 2005; 40: 1785-91.

5.- Kaplan S. Community-acquired methicillinresistant Staphylococcus aureus infections in children. Semin Pediatr Infect Dis 2006; 17 : 113-9.

6.- McDougal L K, Steward C D, Killgore G E, Chaitram J M, McAllister S K, Tenover F C. Pulsed-field gel electrophoresis typing of oxacillin-resistant Staphylococcus aureus isolates from the United States: establishing a national database. J Clin Microbiol 2003; 41: 5113-20.

7.- Purcell K, Fergie J. Epidemic of communityacquired methicillin-resistant Staphylococcus aureus infections: a 14-year study at Driscoll Children's Hospital. Arch Pediatr Adolesc Med 2005; 159: 980-5.

8.- Ochoa T J, Mohr J, Wanger A, Murphy J R, Heresi G P. Community-associated methicillinresistant Staphylococcus aureus in pediatric patients. Emerg Infect Dis 2005; 11: 966-8.

9.- Fridkin S K, Hageman J C, Morrison M, Sanza L T, Como-Sabetti K, Jernigan J A, et al. Methicillin-resistant Staphylococcus aureus disease in three communities. N Engl J Med 2005; 352: 1436-44.

10.- Galeana Villar A. Infección por Staphylococcus aureus meticilino-resistente adquirido en la comunidad. Arch Pediatr Urug 2003; 74: 26-9.

11.- Paganini H, Verdaguer V, Rodríguez A C, Della Latta P, Hernández C, Berberian G, et al. Infecciones causadas por Staphylococcus aureus resistentes a la meticilina en niños provenientes de la comunidad en la Argentina. Arch Argent Pediatr 2006; 104 (4): 293-8.

12.- Mónaco A, Della Latta P, Verdaguer V, Rodríguez C, Hernández C, Pinheiro J, et al. Staphylococcus aureus meticilino-resistente proveniente de la comunidad. estudio de vigilancia en niños de Argentina. V Congreso Argentino de Infectología. Mar del Plata 5-6 de mayo 2007.

13.- Zaoutis T E, Toltzis P, Chu J, Abrams T, Dul M, Kim J, et al. Clinical and molecular epidemiology of community-acquired methicillin-resistant Staphylococcus aureus infections among children with risk factors for healthcare-associated infection: 2001-2003. Pediatr Infect Dis J 2006; 25: 343-8.

14.- Hultén K G, Kaplan S L, González B E, Hammerman W A, Lamberth L B, Versalovic J, et al. Three-year surveillance of community onset healthcare-associated Staphylococcus aureus infections in children. Pediatr Infect Dis J 2006; 25: 349-53.

15.- National Committee for Clinical Laboratory Standards. Performance Standards For Antimicrobial Disk Susceptibility Test: Approved Standard, 7th ed. NCCLS document M2-A7, vol. 10, no.1. Villanova, PA: National Committee for Clinical Laboratory Standards, 2000.

16.- González B E, Martínez-Aguilar G, Hulten K G, Hammerman W A, Coss-Bu J, Avalos-Mishaan A, et al. Severe staphylococcal sepsis in adolescents in the era of communityacquired methicillin-resistant Staphylococcus aureus. Pediatrics 2005; 115: 642-8.

17.- Centers for Disease Control and Prevention. Methicillin-resistant Staphylococcus aureus infections in correctional facilities-Georgia, California, and Texas, 2001-2003. MMWR Morb Mortal Wkly Rep 2003; 52: 992-6.

18.- Purcell K, Fergie J E. Exponential increase in community-acquired methicillin-resistant Staphylococcus aureus infections in South
Texas children. Pediatr Infect Dis J 2002; 21: 988-9.

19.- Said-Salim B, Mathema B, Kreiswirth B N. Community-acquired methicillin- resistant Staphylococcus aureus: an emerging pathogen. Infect Control Hosp Epidemiol 2003; 24: 451-5.

20.- Buckingham S C, McDougal L K, Cathey L D, Comeaux K, Craig A S, Fridkin S K, et al. Emergence of community-associated methicillin-resistant Staphylococcus aureus at a Memphis, Tennessee Children's Hospital. Pediatr Infect Dis J 2004; 23: 619-24.

21.- González B E, Hultén K G, Dishop M K, Lamberth L B, Hammerman W A, Mason E O $\mathrm{Jr}$, et al. Pulmonary manifestations in children with invasive community-acquired Staphylococcus aureus infection. Clin Infect Dis 2005; 41: 583-90.

22.- Como-Sabetti K, Harriman K H, Buck J M, Glennen A, Boxrud D J, Lynfield R, et al. Community-associated methicillin-resistant Staphylococcus aureus: trends in case and isolate characteristics from six years of prospective surveillance. Public Health Rep 2009; 124: 427-35.

23.- Carcillo J A, Castillo L, Thomas J, Kissoon N. Recognizing, understanding, and treating critical community acquired methicillin-resistant Staphylococcus aureus infection in children. Pediatr Crit Care Med 2009; 10: 405-7.

24.- Lee M C, Ríos A M, Aten MF, Mejías A, Cavuoti D, McCracken G H Jr, et al. Management and outcome of children with skin and soft-tissue abscesses caused by communityacquired methicillin-resistant Staphylococcus aureus. Pediatr Infect Dis J 2004; 23: 123-7.

25.- Elliott D J, Zaoutis T E, Troxel A B, Loh A, Keren R. Empiric antimicrobial therapy for pediatric skin and soft-tissue infections in the era of methicillin-resistant Staphylococcus aureus. Pediatrics 2009; 123: e959-66.

26.- Huang J T, Abrams M, Tlougan B, Rademaker A, Paller A S. Treatment of Staphylococcus aureus colonization in atopic dermatitis decreases disease severity. Pediatrics 2009; 123 (5): e80814. 